\title{
The multiple directions of antiferromagnetic spintronics
}

\author{
New developments in spintronics based on antiferromagnetic materials show promise for improved fundamental \\ understanding and applications in technology.
}

\section{T. Jungwirth, J. Sinova, A. Manchon, X. Marti, J. Wunderlich and C. Felser}

S pintronics ${ }^{1-3}$ has revolutionized the field of magnetic recording and it is hoped that it will complement semiconductor-based microelectronics in 'beyond Moore's law' information technologies ${ }^{4}$. Whether designed for sensing, memory or logic applications, spintronic devices have been traditionally based on ferromagnetic materials. Here we present a brief overview of an emerging subfield of spintronics research in which antiferromagnets take the central role ${ }^{5-8}$. We focus on the latest developments, which range from demonstrations of experimental microelectronic memory devices and optical control of antiferromagnetic spins to the interplay of antiferromagnetic spintronics with topological phenomena, noncollinear antiferromagnets, antiferromagnet/ ferromagnet interfaces and synthetic antiferromagnets. We illustrate that the envisaged applications of antiferromagnetic spintronics may expand to areas as diverse as terahertz information technologies or artificial neural networks.

Ferromagnets have been a subject of fascination and have been of great practical value for thousands of years. They were in the cradle of sound recording more than a hundred years ago and carried on through the video recording era to modern datastorage media and computer memories. The latest incarnation of magnetic recording and magnetic sensors, called spintronics, was also all due to ferromagnets?

Antiferromagnets have been known since the 1930s and exhibit the second basic type of magnetic order ${ }^{10}$. They are abundant, but practical means of combining manipulation and detection of antiferromagnetic spins in microelectronic devices have remained elusive until recently ${ }^{11,12}$ (Fig. 1). Among the key prerequisites that, in 2016, led to the experimental demonstration of the antiferromagnetic memory device were studies of relativistic charge-spin coupling phenomena - namely the spin Hall and spin galvanic effects ${ }^{13}$ - that have shaped much of the recent research and development in spintronics ${ }^{14}$.
Combined with broken spatial inversion symmetry, relativistic spin-orbit coupling leads to current-induced spin polarization even in systems with no equilibrium magnetization ${ }^{13}$. Remarkably, this non-equilibrium spin polarization can provide highly efficient means for controlling ordered spins in magnets ${ }^{15}$. While in ferromagnets a global currentinduced spin-polarization has to be induced to couple strongly to the global magnetization $^{16,17}$, antiferromagnets require the generation of a local nonequilibrium spin-polarization whose sign alternates between the lattice sites occupied by opposite magnetic moments ${ }^{18}$. This is achieved, for example, in an antiferromagnetic crystal shown in Fig. 1a, in which the antiparallel magnetic moments occupy locally non-centrosymmetric lattice sites. The resulting staggered current induced spin-orbit field is commensurate with the antiferromagnetic order that allows for the efficient electrical switching ${ }^{11}$.

Over the past year, electrical write/read functionality in experimental memory devices has been verified in semimetallic antiferromagnetic CuMnAs with a Néel temperature of $480 \mathrm{~K}$, deposited by molecular beam epitaxy on silicon and other common semiconductor wafers $^{11,19}$, as well as in sputtered metallic antiferromagnetic films of $\mathrm{Mn}_{2} \mathrm{Au}$ with a Néel temperature above $1,000 \mathrm{~K}\left(\right.$ refs $\left.^{20,21}\right)$. The microfabricated antiferromagnetic bit cells are compatible with standard microelectronic circuitry ${ }^{19}$, are insensitive to magnetic fields and do not generate stray fringing fields ${ }^{11}$. A common feature of antiferromagnetic memory devices is a multilevel switching (Fig. 1b) that allows in principle to integrate memory and logic within the bit cell. It has been observed in antiferromagnetic metal ${ }^{20,21}$, semimetal ${ }^{11,19}$ and semiconductor ${ }^{22}$ structures and associated with multidomain reconfigurations. Moreover, the antiferromagnetic order can also induce the analogue multilevel switching behaviour in an adjacent, exchange-coupled ferromagnetic film ${ }^{23}$.

One of the major attractions of antiferromagnetic spintronics is the prospect of high-speed operation of the devices. Highly reproducible reversible writing by current-induced spin-orbit torque has been demonstrated with writing current pulse length reduced from seconds to picoseconds ${ }^{11,19,24}$. In comparison, the writing speed (the inverse of the writing pulse length) by current-induced spin torques in ferromagnets is limited by the $\mathrm{GHz}$ scale of the ferromagnetic resonance frequency. Beyond this threshold, electrical switching costs a prohibitive amount of energy in ferromagnets ${ }^{25,26}$. On the other hand, the antiferromagnetic resonance is in the $\mathrm{THz}$ range due to the interspin-sublattice exchange, which shifts the accessible electrical writing speeds up by three orders of magnitude compared to ferromagnets ${ }^{18,24,27}$

Laboratory setups with femtosecond lasers are now readily available, allowing the study of optical excitations of antiferromagnets at ultimate speeds. Remarkably, initial studies of ultrafast optical reorientation of spins in antiferromagnets preceded the experimental realization of electrically controlled antiferromagnetic memory devices by a decade. These early optical works took advantage of laser-induced heating that in certain ferrites can trigger a transient change in the antiferromagnetic easy-axis direction $^{28}$. Recently, thermally induced reversible switching between distinct stable states of antiferromagnetic spins was demonstrated in optical experiments in a multiferroic oxide ${ }^{29}$.

However, efficient ultrafast optical control of antiferromagnets requires the utility of non-thermal excitation mechanisms. An example is the recent observation of a large-angle coherent spin-precession in a ferrite antiferromagnet excited by intense terahertz-field transients ${ }^{30}$. Here non-thermal orbital transitions induced a modification of the anisotropy field that drove the antiferromagnetic spins 
a

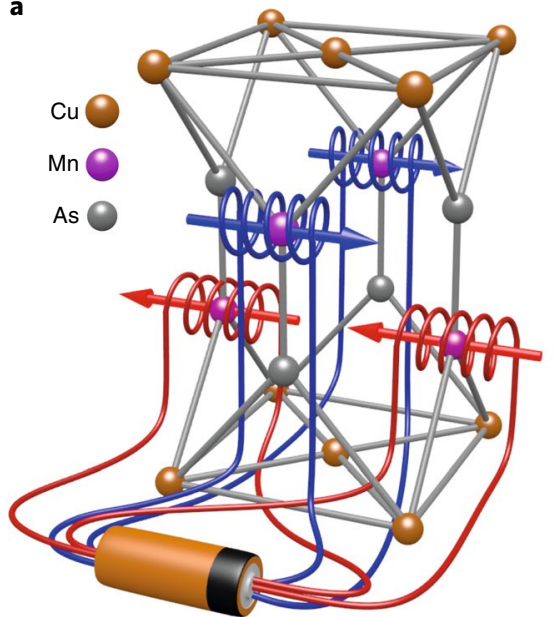

b
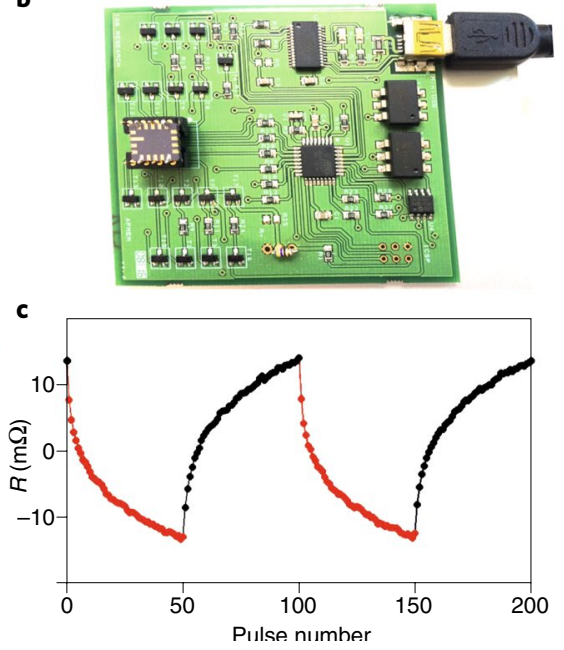

Fig. 1 | Experimental antiferromagnetic memory device. a, Within traditional schemes of magnetic recording, efficient control of antiferromagnets would require the generation of local Ørsted fields of alternating sign, commensurate with the staggered antiferromagnetic order. While winding a microcoil around each individual magnetic atom is unlikely to become a meaningful concept, antiferromagnetic spintronics is discovering new physical principles bringing efficient electrical control of antiferromagnets into reality. b, A memory microchip, placed on a USB printed circuit board, fabricated from CuMnAs antiferromagnet in which a global macroscopic electrical current generates a local spin-orbit field whose sign alternates between the spin sublattices, in analogy to the hypothetical microcoils in a. c, The staggered current-induced spin-orbit field provides efficient means for electrical writing of the multilevel states in the memory bit cell whose design also allows for electrical readout via the antiferromagnetic anisotropic magnetoresistance effect.Credit: adapted from ref. ${ }^{15}$, AIP (a); ref. ${ }^{19}$, Macmillan Publishers Ltd (b); and ref. ${ }^{11}$, AAAS (c)

out of equilibrium. Optical techniques have yet another remarkable feature: the ability to excite not only the uniform antiferromagnetic resonance mode but also higher wave vector modes. This has been illustrated by non-thermal sub-20 fs optical excitation of high-frequency $(20 \mathrm{THz})$ magnons with wavevectors near the edges of the Brillouin zone in a Heisenberg fluoride antiferromagnet ${ }^{31}$.

While the vast majority of optical studies focus on insulating antiferromagnets, a recent trend is to include the same type of (semi)metallic materials used in electrically controlled devices in order to build bridges between electronic and optical sides of the antiferromagnetic spintronics research ${ }^{24,32}$. Connections between electronic and optical tools apply both to the manipulation and detection of antiferromagnetic spins. For example, the frequently employed magneto-optical effects in optical spin detection are the a.c. counterparts of d.c. magnetotransport phenomena used for the electrical readout in the antiferromagnetic memory devices ${ }^{11,32}$.

Recent breakthroughs in efficient electrical and optical manipulation and detection of antiferromagnetic spins unlock a multitude of well-known and newly emerging features of antiferromagnets that are unparalleled in ferromagnets. The $\mathrm{THz}-$ scale spin dynamics is an example of an outstanding property of antiferromagnets acknowledged in the seminal work by Kittel ${ }^{33}$ in the 1950s. This can now be exploited in the research towards magnetic memory and logic devices for the elusive $\mathrm{THz}$ band. Current studies, however, go beyond the uniform antiferromagnetic order by including static and dynamical properties of antiferromagnetic domain walls ${ }^{34-37}$ and more complex spin textures, such as skyrmions $^{38,39}$. In crystal antiferromagnets, these concepts are still mostly at the level of theoretical proposals. However, efficient domain wall and skyrmion motion has been already experimentally demonstrated in synthetic antiferromagnets, which makes these artificial stacks of antiferromagnetically coupled thin-film ferromagnets an attractive basis for the research and development of racetrack memories ${ }^{40,41}$.

A counterexample of a research direction that could have been hardly envisaged at the time of Néel's or Kittel's seminal works is the topological antiferromagnetic spintronics. Topologically protected Dirac, Weyl or Majorana quasiparticles and their interplay with the spintronic control of antiferromagnetic moments are concepts that have been proposed only very recently and still await experimental verification ${ }^{42-45}$. Their utility could be in realizing highly efficient magnetoresitive readout and spin torque writing schemes in antiferromagnetic memories. In a collinear antiferromagnetic Dirac semimetal, for example, a large anisotropic magnetoresistance has been predicted, associated with a topological metal-insulator transition driven by the reorientation of the antiferromagnetic spins $^{43}$. A fundamental feature allowing some antiferromagnets, unlike ferromagnets, to host Dirac band crossings is the effective time-reversal symmetry in which the time reversal combined with other crystal symmetry like spatial inversion is a symmetry operation of the magnetic lattice.

Another avenue for intersection between spintronics and topology-related phenomena opens up in antiferromagnets with more complex non-collinear magnetic orders. For example, several compensated non-collinear antiferromagnets with strong spin-orbit coupling have been shown to exhibit a large anomalous Hall effect even in the absence of a net magnetization of the system ${ }^{46,47}$ (Fig. 2). Here it is the lack of effective time-reversal symmetry in these non-collinear antiferromagnets that allows for the anomalous Hall effect.

Alternatively, chiral non-collinear spin textures can substitute the role of spin-orbit coupling and generate a topological Hall effect in non-coplanar antiferromagnets ${ }^{48}$. Non-collinear antiferromagnets are also predicted to generate spin polarization of the electrical current driven through these crystals, which opens the prospect of realizing analogous phenomena in antiferromagnets to the ferromagnetic giant magnetoresistance and spin-transfer torque ${ }^{49}$.

Research in antiferromagnetic spintronics has so far focused on fundamental physics aspects of this emerging field. Despite its infancy, however, it is timely to frame the field also in the context of its potential future applications. As of 2016, the Moore's lawdriven International Technology Roadmap for Semiconductors is officially at an end ${ }^{4}$. It is being replaced with the new International Roadmap for Devices and Systems in order to tackle the semiconductor scaling problem that is further magnified by the huge increase in the complexity of information technologies. It is envisaged that new material types and new device concepts will join forces with semiconductors in data collection, processing and storage devices. This might help in matching their massive deployment with the required functional diversity and energy efficiency. Robustness against external charge and magnetic 
a

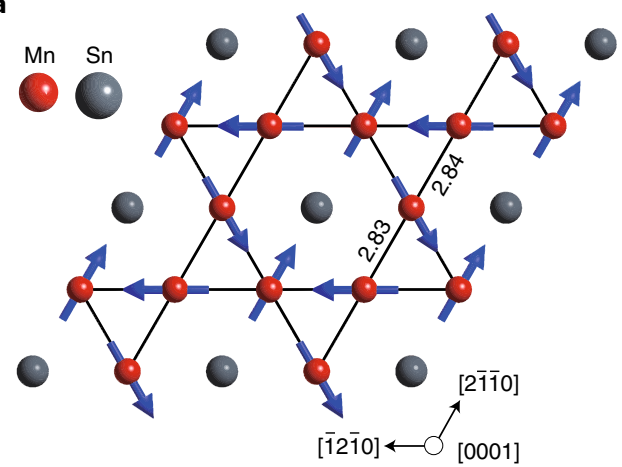

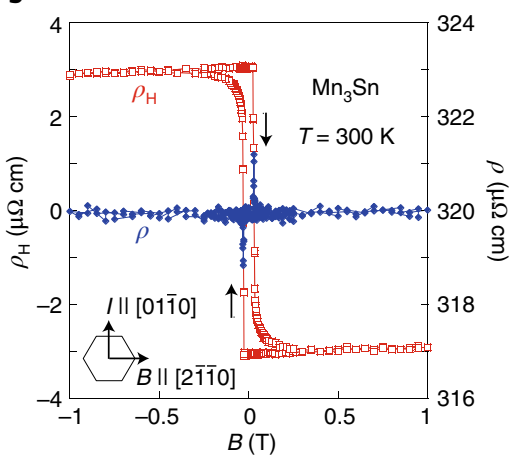

Fig. 2 Antiferromagnetic anomalous Hall effect. a, Non-collinear spin structure in the $a-b$ plane of antiferromagnetic $\mathrm{Mn}_{3} \mathrm{Sn}$. $\mathbf{b}$, Anomalous Hall effect measurement in $\mathrm{Mn}_{3} \mathrm{Sn}$. The chiral spin structure in this non-collinear antiferromagnet allows one to observe the anomalous Hall effect even in the absence of net macroscopic magnetization in the crystal.Credit: adapted from ref. ${ }^{47}$, Macmillan Publishers Ltd

field perturbations, absence of fringing stray fields favourable for high-density integration, non-volatile binary or multilevel bit cells, ultrafast operation, or alternative concepts based on coding information in robust spin textures are examples of the features that might bring antiferromagnetic order into the spotlight of frontier research and development in the beyond-Moore technology era.

The unique merits of antiferromagnetic spintronics can be relevant for several technology fields, including magnetic random access memories (MRAMs), neuromorphic computing and $\mathrm{THz}$ information technology. Antiferromagnetic spin-orbit torque memories can be switched using comparable current densities $\left(\sim 10^{8}\right.$ $A \mathrm{~cm}^{-2}$ ) at the $\mathrm{GHz}$ writing speed to their ferromagnetic spin-orbit torque MRAM counterparts $^{19,25}$. This has been achieved without any systematic optimization of the antiferromagnetic material properties, device designs and physical mechanisms of the employed spin-orbit torques.

Apart from further exploration of the writing schemes, a conceptual breakthrough is required on the readout side of the memory functionality in order to fully exploit the potential of antiferromagnets in MRAMs. Currently, the readout is limited by the several-percent signals typical of the Ohmic anisotropic magnetoresistance ${ }^{11,19-21}$. The scalability of the bit cell size and readout time require significantly larger signals typical of the present ferromagnetic tunnel-magnetoresistance MRAMs ${ }^{3}$. Turning to tunnelling devices might be also a viable route for antiferromagnets $s^{49,50}$ or, alternatively, the anomalous Hall or topological magnetoresistance phenomena might provide the key for devising efficient readout schemes, if compatible with electrical writing ${ }^{43,47,48}$. Another essential and yet virtually unexplored topic is magnetic anisotropy and the associated thermal stability of the memory states in antiferromagnetic materials and structures.

A proof-of-concept study using antiferromagnet/ferromagnet bilayers has already demonstrated the utility of the analogue multilevel switching characteristics in realizing non-volatile programmable synapses for artificial neural networks ${ }^{23}$. Whether using multilevel bit cells made of the antiferromagnet/ferromagnet hybrid structures $^{23}$ or antiferromagnets alone ${ }^{11,19-21}$, the reliability of the associative memory operations will depend on the ability to control the microscopic domain structure of the multilevel cells and to fabricate these cells with highly reproducible analogue characteristics. For these neuromorphic computing applications, optimizing writing, reading and retention will also play an important role.

Another research direction for antiferromagnetic multilevel bit cells is motivated by potential applications in nonvolatile pulse-counter and related memorylogic applications ${ }^{19}$. Highly reproducible pulse-counter characteristics have been demonstrated for up to thousands of pulses with an individual pulse time scaled down from milliseconds to picoseconds $s^{19,24}$. Here, one of the outstanding challenges is the reduction of the pulse delay time along with the individual pulse time in order to realize, within the bit cell, the corresponding memory-logic operation at frequencies scaled up to the $\mathrm{GHz}$ or even $\mathrm{THz}$ range. These considerations connect antiferromagnetic spintronics to future developments in the field of $\mathrm{THz}$ information and communication technologies. Antiferromagnets are favourable candidate materials not only for the $\mathrm{THz}$ memory-logic devices but also for other essential components, including $\mathrm{THz}$ sources or detectors.

T. Jungwirth ${ }^{1,2 \star}$, J. Sinova ${ }^{1,3}$, A. Manchon ${ }^{4}, X$. Marti ${ }^{1,5}$, J. Wunderlich ${ }^{1,6}$ and C. Felser ${ }^{7}$ ${ }^{1}$ Institute of Physics, Academy of Sciences of the Czech Republic, Praha, Czech Republic. ${ }^{2}$ School of Physics and Astronomy, University of Nottingham, Nottingham, UK. ${ }^{3}$ Institut für Physik, Johannes Gutenberg Universität Mainz, Mainz, Germany. ${ }^{4}$ Physical Science and Engineering Division, King Abdullah University of Science and Technology (KAUST), Thuwal, Saudi Arabia. ${ }^{5}$ IGS Research, Calle La Coma, Tarragona, Spain. ${ }^{6}$ Hitachi Cambridge Laboratory, Cambridge, UK. ${ }^{7}$ Max Planck Institute for Chemical Physics of Solids, Dresden, Germany.

*e-mail: jungw@fzu.cz

Published online: 2 March 2018

https://doi.org/10.1038/s41567-018-0063-6

References

1. Chappert, C., Fert, A. \& Van Dau, F. N. Nat. Mater. 6 , 813-823 (2007).

2. Brataas, A., Kent, A. D. \& Ohno, H. Nat. Mater. 11, 372-381 (2012)

3. Kent, A. D. \& Worledge, D. C. Nat. Nanotech. 10, 187-191 (2015)

4. Waldrop, M. M. Nature 530, 144-147 (2016).

5. MacDonald, A. H. \& Tsoi, M. Philos. Trans. R. Soc. A 369, 30983114 (2011).

6. Gomonay, E. V. \& Loktev, V. M. Low Temp. Phys. 40, $17-35$ (2014).

7. Jungwirth, T., Marti, X., Wadley, P. \& Wunderlich, J. Nat. Nanotech. 11, 231-241 (2016).

8. Baltz, V. et al. Preprint at https://arxiv.org/abs/1606.04284 (2016)

9. Wolf, S. A. et al. Science 294, 1488-1495 (2001)

10. Néel, L. in Nobel Lectures, Physics 1963-1970 318-341 (Elsevier, Amsterdam, 1972)

11. Wadley, P. et al. Science 351, 587-591 (2016).

12. Marrows, C. Science 351, 558-559 (2016).

13. Sinova, J., Valenzuela, S. O., Wunderlich, J., Back, C. H. \& Jungwirth, T. Rev. Mod. Phys. 87, 1213-1260 (2015).

14. Pulizzi, F. Nat. Mater. 11, 367 (2012)

15. Sinova, J. \& Jungwirth, T. Phys. Today 70, $38-42$ (July, 2017).

16. Miron, I. M. et al. Nature 476, 189-193 (2011).

17. Liu, L. et al. Science 336, 555-558 (2012)

18. Železný, J. et al. Phys. Rev. Lett. 113, 157201 (2014).

19. Olejník, K. et al. Nat. Commun. 8, 15434 (2017).

20. Bodnar, S. Yu. et al. Nat. Commun. 9, 348 (2018).

21. Meinert, M., Graulich, D. \& Matalla-Wagner, T. Preprint at https://arxiv.org/abs/1706.06983 (2017).

22. Kriegner, D. et al. Nat. Commun. 7, 11623 (2016).

23. Borders, W. A. et al. Appl. Phys. Express 10, 013007 (2017).

24. Olejnik, K. et al. Preprint at https://arxiv.org/abs/1711.08444 (2017).

25. Garello, K. et al. Appl. Phys. Lett. 105, 212402 (2014).

26. Prenat, G. et al. IEEE Trans. Multi-Scale Comput. Sys 2, 49-60 (2016).

27. Roy, P., Otxoa, R. M. \& Wunderlich, I. Phys. Rev. B 94, 014439 (2016).

28. Kimel, A. V., Kirilyuk, A., Tsvetkov, A., Pisarev, R. V. \& Rasing, T. Nature 429, 850-853 (2004).

29. Manz, S. et al. Nat. Photon. 10, 653-656 (2016)

30. Baierl, S. et al. Nat. Photon. 10, 715-718 (2016).

31. Bossini, D. et al. Nat. Commun. 7, 10645 (2016).

32. Saidl, V. et al. Nat. Photon. 11, 91-97 (2017).

33. Kittel, C. Phys. Rev. 82, 565-565 (1951).

34. Hals, K. M. D., Tserkovnyak, Y. \& Brataas, A. Phys. Rev. Lett. 106, 107206 (2011).

35. Gomonay, O., Jungwirth, T. \& Sinova, J. Phys. Rev. Lett. 117, 017202 (2016).

36. Shiino, T. et al. Phys. Rev. Lett. 117, 087203 (2016).

37. Selzer, S., Atxitia, U., Ritzmann, U., Hinzke, D. \& Nowak, U. Phys. Rev. Lett. 117, 107201 (2016) 
38. Zhang, X., Zhou, Y. \& Ezawa, M. Sci. Rep. 6, 24795 (2016).

39. Barker, J. \& Tretiakov, O. A. Phys. Rev. Lett. 116, 147203 (2016).

40. Yang, S.-H., Ryu, K.-S. \& Parkin, S. Nat. Nanotech. 10,

221-226 (2015).

41. Zhang, X., Ezawa, M. \& Zhou, Y. Phys. Rev. B 94, 064406 (2016)

42. Tang, P., Zhou, Q., Xu, G. \& Zhang, S.-C. Nat. Phys. 12, $1100-$ 1104 (2016).

43. Šmejkal, L., Železný, J., Sinova, J. \& Jungwirth, T. Phys. Rev. Lett. 118, 106402 (2017).

44. Wang, Z. F. et al. Nat. Mater. 15, 968-973 (2016).
45. Yang, H. et al. New J. Phys. 19, 015008 (2017).

46. Chen, H., Niu, Q. \& MacDonald, A. H. Phys. Rev. Lett. 112 017205 (2014).

47. Nakatsuji, S., Kiyohara, N. \& Higo, T. Nature 527 212-216 (2015).

48. Sürgers, C., Fischer, G., Winkel, P. \& Löhneysen, H. V. Nat. Commun. 5, 3400 (2014).

49. Železný, J., Zhang, Y., Felser, C. \& Yan, B. Phys. Rev. Lett. 119 , 187204 (2017).

50. Park, B. G. et al. Nat. Mater. 10, 347-351 (2011).

\section{Acknowledgements}

The authors acknowledge the EU FET Open RIA Grant No. 766566, the Alexander von Humboldt Foundation, the Transregional Collaborative Research Center (SFB/TRR) No. 173 SPIN+X, the Grant Agency of the Czech Republic Grant No. 14-37427G, the Ministry of Education of the Czech Republic Grant Nos LM2015087 and LNSM-LNSpin, the ERC Synergy Grant No. 610115, and the King Abdullah University of Science and Technology Grant No. OSR-2015-CRG4-2626.

\section{natureresearch EDITING SERVICE}

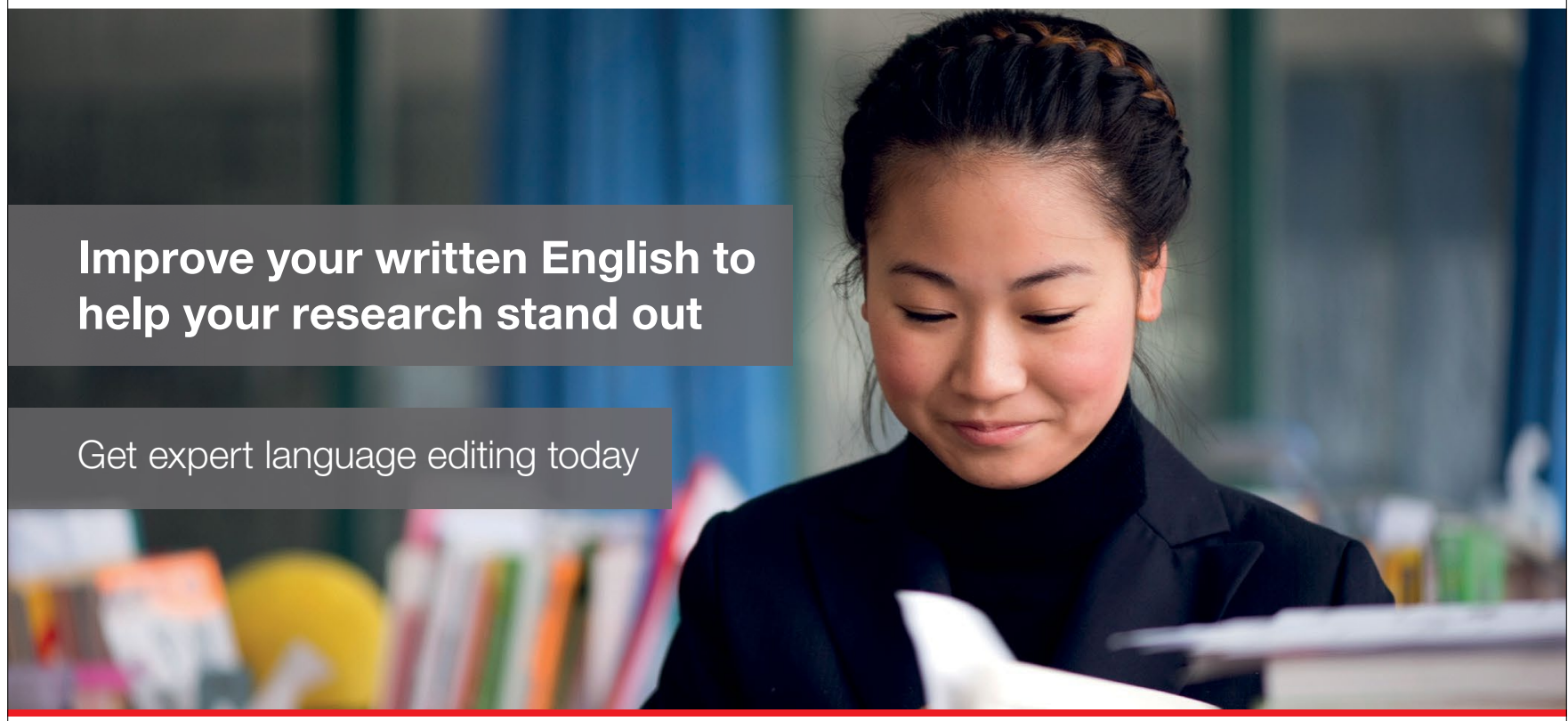

authorservices.springernature.com/language-editing

Formerly known as NPG Language Editing 